\title{
A Special Section on X-ray and Extreme Ultraviolet Multilayer Coatings
}

Soft X-ray and Extreme Ultraviolet (EUV) radiation (defined as light with wavelength in the 1 to $100 \mathrm{~nm}$ range) has been playing a growing role in major scientific and industrial applications over the last 20 years: astrophysics and solar physics, materials sciences, biology, semiconductor industry, plasma diagnostics. Especially the emergence of novel EUV/X-ray sources with unprecedented brightness and coherence (4th generation synchrotrons, free-electron lasers, tabletop lasers, high-harmonic generation and attosecond sources) has ushered a new era in the fields of materials science, chemistry, plasma physics, biology and life sciences. Several scientific and technological breakthroughs have been achieved recently. X-ray radiation from the SPRING-8 synchrotron light source in Japan has been focused on a spot with diameter smaller than $10 \mathrm{~nm}$; this constitutes the smallest spot of light ever produced and could enable studies with the most exquisite spatial resolution. The generation of ultrashort EUV pulses with a duration less than 0.1 femtosecond (= 100 attoseconds) made it possible for the first time to observe electron orbitals in molecules. Photolithography scanners based on EUV light have entered the world of semiconductor manufacturing in order to produce the next generation of computer chips. In 2007, the EUV telescopes aboard NASA's Solar Terrestrial Relations Observatory (STEREO) mission provided the first 3D images of the solar corona with its protuberances. Furthermore, since 2010, the Solar Dynamics Observatory (SDO), NASA's most advanced solar mission, has been continuously transmitting full-disk images of the solar corona every 10 seconds, simultaneously at 7 EUV wavelengths with 1 arcsec resolution. Observing the Sun with such unprecedented temporal and spatial resolution has led to groundbreaking discoveries related to the Sun's extremely complex magnetic field and has led to better understanding of extreme events such as solar flares and coronal mass ejections, which can seriously affect earth and space environments.

These breakthroughs have been enabled by the development of new optical components dedicated to the EUV/ $\mathrm{X}$-ray spectral range. The main challenges in fabricating
EUV/X-ray optics come from the properties of materials in this spectral range. With the refractive index of any material being very close to 1 , the refractive phenomena at any interface are weak and the reflectivity of any single material at non-grazing incidence angles is near zero. Moreover, all materials strongly absorb X-rays, so the transmission of any component is almost zero. Multilayer interference mirrors are enabling optical components in most EUV/X-ray optical systems. Demonstrated experimentally for the first time in the 1970 s by E. Spiller, these mirrors consist of periodic or aperiodic structures of alternating thin film layers of 2 or more materials with nanometer-scale thickness, deposited on an optical substrate. The constructive interference between the layers results in efficient reflectance at EUV/X-ray wavelengths even at near-normal incidence angles thus enabling the operation of a wide range of optical components including imaging and illumination systems, reflective filters, grating spectrometers and polarizers.

However, the fabrication of high-performance multilayer coatings poses serious technical challenges. Multilayer coatings need to obey several stringent (and often conflicting) requirements, including: (i) contain materials with good optical contrast in the wavelength region of operation (ii) contain tens or hundreds of layers with nanometer-scale thickness, where each layer is deposited with picometer-scale precision (iii) form stable layer interfaces with minimal interdiffusion and with smoothness on the order of the atomic dimension, (iv) have low thin film stress, (v) have stable reflective performance for periods up to 10 years, or even longer.

This special section aims to provide a survey of current topics and major development lines in the very active research area of X-ray and EUV multilayer coating development. It gathers studies of the properties of these nanoscale structures (microstructure, mechanical stress, lifetime stability) with a focus on new promising material combinations and on short-period multilayers, with individual layer thicknesses around (or less than) $1 \mathrm{~nm}$. Technological developments, new characterization 
methods and applications are also addressed in this special section.

\author{
Guest Editors \\ Franck Delmotte \\ Institut d'Optique Graduate School \\ Palaiseau \\ France
}

Maria Guglielmina Pelizzo

National Research Council of Italy

Institute for Photonics and Nanotechnologies

Padova

Regina Soufli

Lawrence Livermore National Laboratory

Livermore

California

\section{ABOUT THE GUEST EDITORS}
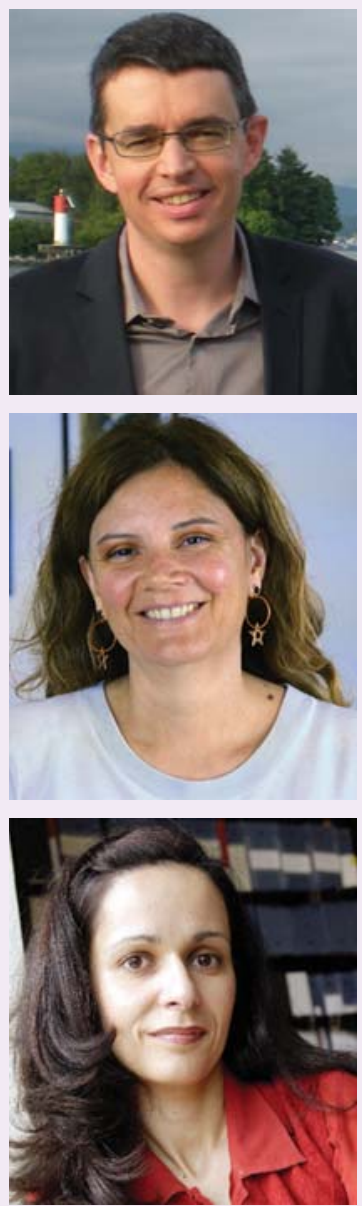

Franck Delmotte obtained a Ph.D. in Electrical Engineering at the University Paris-Sud (Orsay, France) in 1998. He is currently Professor at the Institut d'Optique Graduate School where he is teaching Optical Thin Films and X-ray Optics. Head of the XUV Optics Group at Laboratoire Charles Fabry, his main research interests consist of interference coatings for extreme ultraviolet (EUV) and X-ray applications including EUV telescopes for space science, X-ray diagnostics for laser plasma experiments (Laser Mega Joule), optical components for attosecond pulses and free electron lasers, multilayer gratings for synchrotron radiation. He is currently Co-Investigator of the Extreme UV Imagers consortium for the Solar Orbiter ESA mission, in charge of the development and delivery of mirrors and coatings for the two EUV telescopes.

Maria Guglielmina Pelizzo received her Ph.D. in Space Science and Technology from the University of Padova, Italy, in 2000. She is currently a researcher of the National Research Council of Italy-Institute for Photonics and Nanotechnologies and adjunct professor of Optics and Photonics at University of Padova. Her research is focused on optical thin films and she has been the responsible scientist on various programs in the field of extreme ultraviolet optics for space and free electron laser applications. She is currently the principal investigator of an ESA project devoted to the study of materials and coatings operating in harsh space environment. She is Co-investigator of Metis coronagraph on board of ESA Solar Orbiter, of Phebus spectrometer on board of ESA Bepi-Colombo, and of the Janus camera on board of ESA Juice.

Regina Soufli received her Ph.D. in Electrical Engineering from the University of California, Berkeley, in 1997. At Lawrence Livermore National Lab she has been principal investigator on programs related to extreme ultraviolet (EUV) and X-ray optics for semiconductor lithography, solar physics and astrophysics, synchrotron and free-electron lasers, and high-energy physics. She recently developed optics for the Linac Coherent Light Source, the world's first X-ray free electron laser, and EUV multilayer optics for NASA's Solar Dynamics Observatory and GOES space weather satellites. She is a Fellow of the Optical Society (OSA) and a Senior Member of SPIE. She was granted a "Jean d'Alembert" fellowship by Université Paris-Saclay and is currently a visiting researcher at the Institut d'Optique Graduate School/Laboratoire Charles Fabry. 